\title{
MALLA DE POLIPROPILENO MULTIFILAMENTO CON AUTOFIJACIÓN DE SILICONA EXTERIORIZADA A VAGINA
}

\author{
Vicente Solà Dalenz, Jack Pardo Schanz, Paolo Ricci Arriola y Enrique Guiloff Fische.
}

Unidad de Uroginecología y Cirugía Vaginal. Departamento de Ginecología y Obstetricia. Clínica Las Condes. Santiago. Chile.

\begin{abstract}
Resumen.- OBJETIVO: Describir un caso de falla de en una cirugía de cinta suburetral para corrección de la incontinencia de orina de esfuerzo con sistema Safyre VS (vaginal-suprapubic approach) multifilamento, a los 34 meses de buena evolución.

MÉTODO: Descripción del caso y su resolución.

RESULTADO: Se presentó con descenso del sistema de sujeción lateral y erosión de la mucosa vaginal con exposición de la cinta e infección. Se retiró con gran facilidad el sistema de anclaje de silicona que se encontraba suelto y se colocó una nueva cinta suburetral con sistema TVT-O, recuperando la continencia antes lograda.
\end{abstract}

CONCLUSIONES: La malla sintéticas de los sistemas de corrección con cinta suburetral para la incontinencia de orina de esfuerzo, deben ser de polipropileno, macroporo y monofilamento. Estas características permiten una mejor biointegración, reduciendo teóricamente el número de complicaciones (erosión de pared vaginal, exposición y sobreinfección). Respecto a la falla del sistema de sujeción de silicona, ya que no se han informado otros casos en los estudios de seguimiento entre 12 y 36 meses (media 14 y 18 meses), sólo los estudios que contemplen seguimientos a largo plazo permitirán decir si se trata de un caso aislado o aparecerán otros.

Palabras clave: Safyre. Safyre VS. TVT-O. Incontinencia urinaria de esfuerzo. Malla multifilamento. Malla monofilamento.

Summary.- OBJECTIVES: To describe the failure of one case of suburethral tape for the correction of the stress urinary incontinence with the multifilament Sapphire VS (vaginal-suprapubic approach) system after 34 months of good outcome.

METHODS: Description of the case and its resolution.

RESULTS: The patient presented with a descent of the lateral fixation and erosion of the vaginal mucosa with exposure of the mesh and infection. The silicone fixation system, which was loose, was retrieved very easily and a new suburethral mesh with the system TVT-O was inserted; the patient recovered continence as before.

CONCLUSIONS: Synthetic mesh in the suburethral tape systems for stress urinary incontinence correction should be polypropylene, macropore and monofilament. These characteristics enable better bio integration, theoreti- 
cally reducing the number of complications /vaginal wall erosion, exposition and over infection). In relation with the failure of the silicone fixation system, no other casers have been reported in follow-up studies between 12 and 36 months (mean follow-up 14 and 18 months), so hat studies with long-term follow-up will be able to determine if this is an isolated case or there will be others.

Keywords: Sapphire. Sapphire VS. TVT-O. Stress urinary incontinence. Multifilament mesh. Monofilament mesh.

\section{INTRODUCCIÓN}

Múltiples son las técnicas quirúrgicas que se han utilizado para la corrección de la Incontinencia de Orina de Esfuerzo (IOE). Lejos parecen los tiempos en que se usaron las técnicas de plicatura, como la de Kelly (1913), Stockel (1921) y Marion (1935). Más tarde se utilizó la operación de Marshall, Marchetti y Krantz (1949), hasta llegar a la técnica de Burch (1961) (1), que durante muchos años se consideró como la mejor para la corrección de la IOE.

Sin embargo, con el desarrollo de la teoría de la hamaca suburetral durante la década de los noventa, se comenzaron a utilizar las técnicas quirúrgicas con cintas suburetrales libres de tensión. El TVT descrito por Ulmsten (1996) es el primero de estos sistemas, cuya principal desventaja es que requiere una cistoscopia intraoperatoria. Otro sistema con los mismos principios es el Safyre VS (2), que a diferencia del TVT, posee a ambos lados de la cinta un brazo de autofijación de silicona. Más tarde en un intento por simplificar la técnica, Delorme (2001), describe el TOT (trans-obturator-tape) y luego Jean de Leval (2003) el TVT-O.

\section{CASO CLÍNICO}

Paciente de 52 años, con antecedente de histerectomía total por vía abdominal realizada en el 2000, a partir de esa fecha con tratamiento hormonal. En marzo del 2003 se indica cirugía por incontinencia de orina de esfuerzo. Se escoge en esa ocasión una cirugía de sling con Safyre VS (vaginalsuprapubic approach, Promedon, Cordoba, Argentina), el que se reajustó a las 24 horas por detectarse sobrecorrección. La paciente a partir de esta fecha y hasta enero del 2006 relata haberse mantenido con normalidad respecto a su continencia urinaria, manteniendo los buenos resultados observados después de la cirugía. A partir de esta fecha comienza con escapes de orina progresivos, hasta presentarse frente a mínimos esfuerzos. Por este motivo decide consultar en nuestra Unidad de Uroginecología y Cirugía Vaginal en enero del presente año.

En la anamnesis se obtuvo la historia ya descrita y además de encontrarse con una incontinencia de orina de esfuerzo, refirió disuria. Al examen vaginal destacaba la presencia de flujo vaginal amarillo y de muy mal olor. En la mucosa vaginal de la porción suburetral se observaba la exposición de una malla de 3,5 por 1 centímetro (Figura 1). Se detectó al exa-

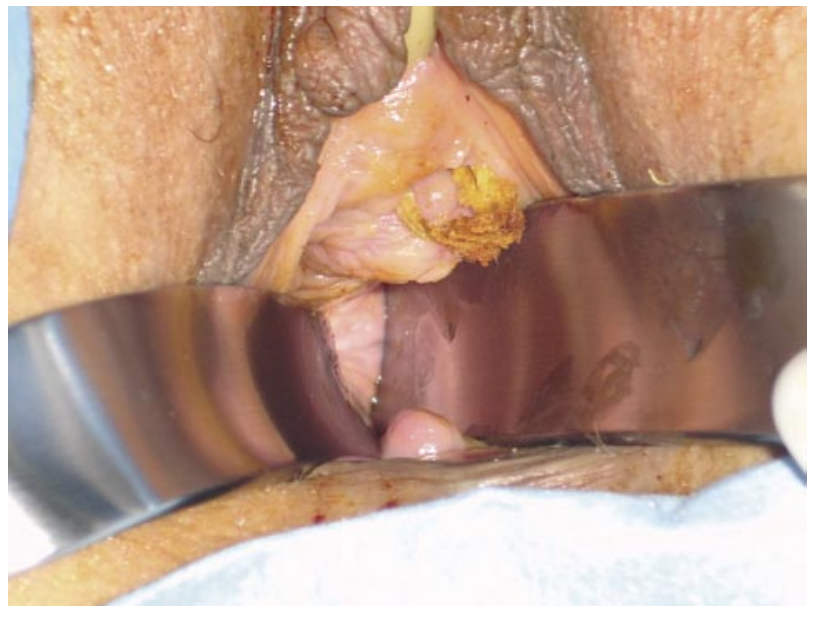

FIGURA IA. Exteriorización de la malla en mucosa vaginal.

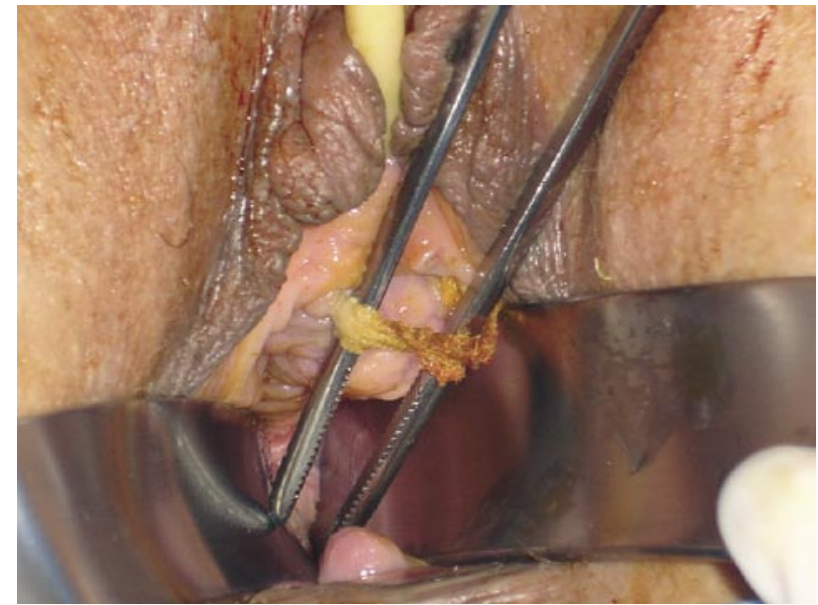

FIGURA IB. Detalle de malla exteriorizada en mucosa vaginal, en su porción suburetral. 
TABLA I. CLASIFICACIÓN POP-Q.

\begin{tabular}{|l|l|l|}
\hline$+1 \mathrm{Aa}$ & $-3 \mathrm{Bb}$ & $-7 \mathrm{C}$ \\
\hline $4 \mathrm{Gh}$ & $2 \mathrm{~Pb}$ & $8 \mathrm{Tvl}$ \\
\hline$+1 \mathrm{Ap}$ & $-3 \mathrm{Bp}$ & $-\mathrm{D}$ \\
\hline
\end{tabular}

Prolapso grado Il según la clasificación POP-Q.
En un segundo tiempo, después de seis semanas se realizó una segunda cirugía para corrección de su incontinencia de orina de esfuerzo. Esta vez se realizó un TVT-O (Johnson \& Johnson) con técnica clásica (3). Además se corrigió tanto el prolapso anterior como el posterior, reforzando la fascia en ambos casos con técnica con malla Gynemesh PS (Gynecare, Johnson \& Johnson) libre de tensión (4). La paciente actualmente permanece con su patología de incontinencia solucionada ("paciente seca").

\section{DISCUSIÓN}

Se trata de un caso de falla de un sistema men ginecológico un cistocele y rectocele. Prolapso genital grado II, utilizando el sistema de cuantificación POP-Q (Pelvic Organ Prolapse Quantification) de la ICS (International Continence Society) (Tabla I). Se realizó un $Q$ tip test que fue positivo (30 grados).

Se realizó examen bioquímico y cultivo de orina, en que se certificó una infección urinaria por escherichia coli.

Se indicó tratamiento antibiótico con ciprofloxacino 500 mgs cada 12 horas y metronidazol 500 mgs cada 8 horas, ambos vía oral. Se planificó el retiro del implante en pabellón. A la inspección se pudo constatar que los brazos laterales de silicona que constituían el sistema de sujeción, se encontraban desplazados hacia abajo, además de sueltos, y la cinta expuesta en vagina. Se retiró la cinta y los implantes laterales con gran facilidad, casi sin necesidad de tracción (Figuras 2 y 3 ).

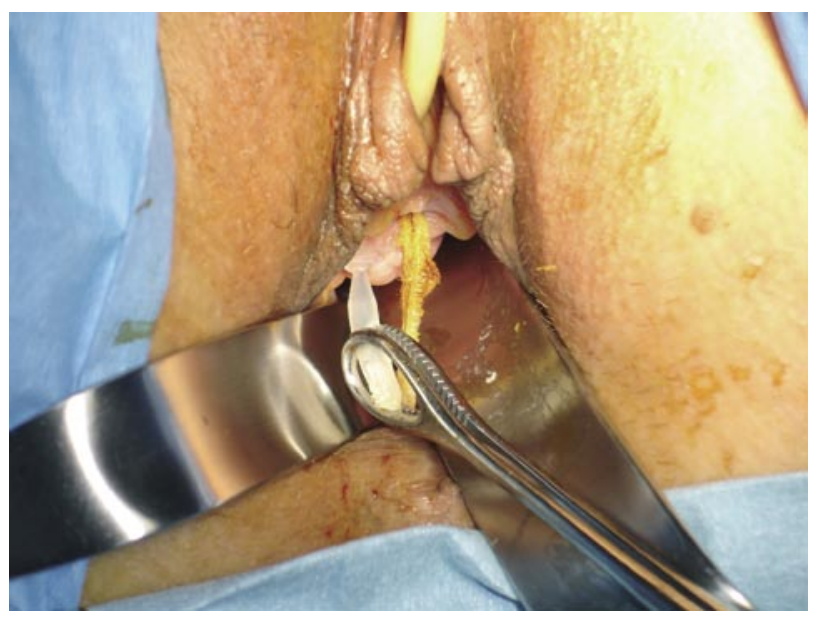

FIGURA 2. Extracción en pabellón de sistema Safyre.

Secuencia que muestra la extracción con gran facilidad de sistema Safyre, no se constata adhesión del sistema de sujeción lateral (brazos de silicona). 
tamaño del poro, que junto al tipo de material y la trama de los filamentos, otorgan el grado de flexibilidad. Estas características son de gran importancia, ya que estas depende la potencial infección, biointegración y erosión de la vagina. Los poros deben tener un tamaño suficiente para permitir el paso de leucocitos (9 a $15 \mu$ ) y macrófagos (16 a $20 \mu$ ) para reducir el riesgo de infección. Las mallas multifilamentos presentan espacios entre los filamentos de menos de $10 \mu$, permitiendo el paso de bacterias (1 $\mu$ ) y no el de macrófagos y leucocitos, lo que facilita la infección. (8) Las mallas de polipropileno, monofilamento y macroporo $(75 \mu)$, clasificadas dentro de las Tipo I (según la clasificación de prótesis quirúrgicas) (9), cumplen con las características deseables a la luz de los conocimientos actuales, para reducir el riesgo de complicaciones, al lograr una mejor biointegración.

Por otro lado no podemos dejar de mencionar que la infección de la malla, facilitada seguramente en este caso por tratarse de una malla multifilamento, es una de las principales causas de erosión de la pared vaginal. Erosión que además puede haber sido facilitada por el descenso de la malla desde su localización suburetral.

En lo referente a la facilidad con que se retiró el sistema, llama la atención la escasa adherencia que mostraba en este caso el brazo de sujeción lateral de silicona (polidimetilsiloxano). Lo que no ha sido informado por otras publicaciones con seguimientos entre 12 y 36 meses, con medias de 14 y 18 meses $(5,6)$. Sin embargo debemos recordar que la silicona no se biointegra, por lo que la mantención de este sistema se basa en primer lugar en una sujeción otorga-

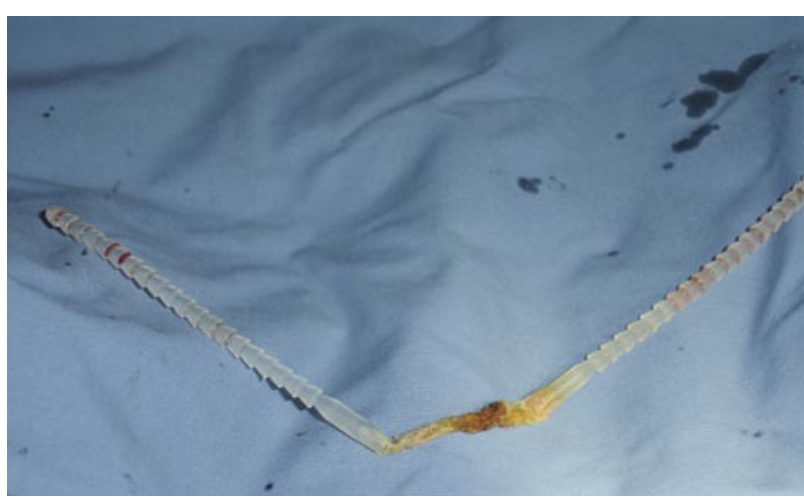

FIGURA 3. Sistema Safyre con malla multifilamento extraido.

Se observa el sistema Safyre extraído completamente. da sólo por una compresión mecánica de las diferentes estructuras corporales que permanecen alrededor (músculos y grasa principalmente) y la formación de una seudocápsula. Y en segundo lugar, cada brazo lateral está formado por la unión de un conjunto de estructuras de forma cónica de base inferior, lo que otorga un efecto también mecánico, que evita el deslizamiento del brazo de silicona. Si lo comparamos con los sistemas en que los brazos laterales están formados por la misma malla suburetral, estos últimos, al menos teóricamente, debieran otorgar un sistema de mejor sujeción. Ya que al igual que la malla en su porción suburetral, también estarán expuestos al fenómeno de biointegración, otorgando una teórica mejor sujeción y que podría mejorar o aumentar en el tiempo.

La resolución de este caso además contempló la corrección de cistocele y rectocele que según lo informado no se encontraban en la primera cirugía de IOE. Por lo que si lo analizamos teóricamente desde el punto de vista de la teoría integral de la continencia, permite deducir que existió un avance en la alteración del suelo pélvico, expresándose como prolapso y que seguramente de igual forma continuó alterando también las estructuras de sostén suburetrales. Por otro lado, es posible que ante esta nueva condición de avance de las alteraciones del piso pélvico, el sistema de sujeción de la malla multifilamento, no fue capaz de mantener su posición, por no tener biointegración, terminando por ceder.

Específicamente respecto al sistema Safyr, hoy se encuentra disponible el Safyr VS (sistema tipo TVT) y Safyr T (Tipo TOT) (10), ambos con mallas de polipropileno, macroporo, monofilamento.

\section{CONCLUSIÓN}

Los diferentes sistemas para la corrección quirúrgica de la incontinencia de orina de esfuerzo con malla, deben utilizar las de polipropileno, macroporo y monofilamento. Ya que es la malla que probadamente por los diferentes estudios, ofrece la mejor biointegración, permitiendo reducir el número de complicaciones (erosión de pared vaginal, exposición y sobreinfección).

Respecto a la falla del sistema de sujeción de silicona, ya que no se han informado otros casos en los estudios de seguimiento entre 12 y 36 meses (media 14 y 18 meses), sólo los estudios que contemplen seguimientos a largo plazo permitirán decir si se trata de un caso aislado o aparecerán otros. 


\section{BIBLIOGRAFÍA y LECTURAS RECOMENDADAS ( ${ }^{*}$ lectura de interés $y^{* *}$ lectura fundamental)}

1. BURCH, J.C.: "Urethrovaginal fixation to Cooper's ligament for correction of stress incontinence, cystocele, and prolapse". Am. J. Obstet. Gynecol., 81: 281, 1961.

**2. PALMA, P.C.; RICCETTO, C.L.; DAMBROS, M. y cols.: "A readjustable minimally invasive sling for female urinary stress incontinence". Int. Braz. J. Urol., 29: 353, 2003.

*3. SOLÀ DALENZ, V.; PARDO SCHANZ, J.; RICCI ARRIOLA, P. y cols.: "Minimal invasive surgery in female urinary incontinence: TVT-O". Actas Urol. Esp., 30: 61, 2006.

4. SOLÀ DALENZ, V.; PARDO SCHANZ, J.; RICCI ARRIOLA, P. y cols.: "Tension free monofilament polypropylene mesh in cystocele and rectocele concomitant repair". Actas Urol. Esp., 29: 977, 2005.

**5. PALMA, P.C.; RICCETTO, C.L.; DAMBROS, M. y cols.: "A new concept for adjustable minimally invasive sling for female urinary stress incontinence". Actas Urol. Esp., 28: 749, 2004.

**6. PALMA, P.C.; DAMBROS, M.; RICCETTO, C.L. y cols.: "The Ibero-American experience with a re-adjustable minimally invasive sling". BJU Int., 95: 341, 2005.

7. HERMIEU, J.F.; MILCENT, S.: "Synthetic suburethral sling in the treatment of stress urinary incontinence in women". Prog. Urol., 13: 636, 2003.

**8. BAFGHI, A.; BENIZRI, E.I.; TRASTOUR, C. y cols.: "Multifilament polypropylene mesh for urinary incontinence: 10 cases of infections requiring removal of the sling". BJOG., 112: 376, 2005.

9. BIRCH, C.; FYNES, M.M.: "The role of synthetic and biological prothesis in reconstructive pelvic floor surgery”. Curr. Opin. Gynecol., 14: 527, 2002.

**10. PALMA, P.; RICCETTO, C.; HERRMANN, V. y cols.: "Transobturator SAFYRE sling is as effective as the transvaginal procedure". Int. Urogynecol. J. Pelvic Floor Dysfunct., 16: 487, 2005. 